\title{
Pulsed-waveform generator based on coupled oscillators
}

\author{
Mabel Pontón, Almudena Suárez \\ University of Cantabria, Santander, Spain
}

\begin{abstract}
Waveform sharpening in nonlinear transmission lines (NLTL) is analyzed in detail and applied to the design of a pulse-generator based on two coupled oscillators. The maximum propagation frequency through the NLTL is calculated with a realistic numerical technique, which enables an optimized design. By coupling two NLTL oscillators terminated in grounded stubs of different length, three design parameters are available, which enables a flexible mechanism for pulse formation. A reducedorder model is proposed for the efficient determination of the tuning voltage in one of the oscillator elements. The analysis and design techniques have been applied to a prototype at $0.8 \mathrm{GHz}$.

Index Terms - Shock wave, nonlinear transmission line, oscillator.
\end{abstract}

\section{INTRODUCTION}

Nonlinear-transmission lines (NLTL) exhibit strong harmonic-generation capabilities, which enable the implementation of frequency multipliers of high order [1]. They can also are used in sampling-instrumentation systems and ultrawideband sensors [2-3]. In an NLTL composed by inductor-varactor cells, the propagation delay decreases with the reverse voltage, due to diode variable capacitance [3-5]. In an ideal continuous medium, the nonlinear capacitance would lead to the formation of a shockwave when the high-amplitude section of the waveform overtakes the bottom [3]. To clarify the concept, the ideal continuous line, without loss, is modelled as:

$$
\frac{\partial v}{\partial x}=-l \frac{\partial i}{\partial t} ; \frac{\partial i}{\partial x}=-c(v) \frac{\partial v}{\partial t}
$$

where $l$ and $c(v)$ are the inductance and capacitance per unit length. Assuming a matched termination, the voltage can be expressed as $v(s)$, where $s=x-1 / \sqrt{l c(v)} t=x-g(v) t$, and one can derive an analytical expression for $\partial v / \partial t$ :

$$
\frac{\partial v}{\partial t}=-\frac{\partial v}{\partial s} g(v)\left[1+\frac{\partial v}{\partial s} \frac{\partial g(v)}{\partial v} t\right]^{-1}
$$

Infinite slope points would be obtained at time values where the term in brackets is equal to zero. In practice, the NLTL is a lossy and dispersive network, the latter due its discrete nature, which will limit the propagation frequency. However, the waveform transition times can be minimized through a suitable design, taking into account the inductance and varactor-diode characteristics and the number of cells. The line Bragg frequency $f_{B}$ in linear operation is $f_{B}=1 /\left(\pi C_{o} L\right)$, with $C_{o}$ the small-signal capacitance. Under nonlinear operation, the Bragg frequency increases due to effect of the nonlinear capacitances. The NLTL admits no analytical solution and approximate expressions for this frequency have been provided [4-5], based on the replacement of $C_{o}$ by the saturated capacitance or by an average capacitance [5]. These expressions neglect the impact of the loss resistance and assume equal minima and maxima of the voltage waveform across the NLTL cells. Here an efficient numerical technique for a realistic calculation of the maximum propagation frequency is presented. Using the sharpening mechanism associated to the shockwave formation, an autonomous pulse generator is proposed. It consists of two coupled oscillators from which the output pulse is extracted in a differential manner. Compared with the soliton-based oscillators [6-7], it enables more flexibility in the pulse forming, which relies on the phase shift between the oscillator elements and the two different oscillator loads. Indeed, the soliton has a pulse width which is inversely proportional to the pulse amplitude, so both magnitudes are linked when using this concept. Monocycle pulses, with a spectrum that does not contain low frequency components, can also be generated as particular cases.

\section{SHOCKWAVE GENERATOR}

In a first stage, an NLTL has been designed based on the varactor diode SMV1232. The diode cut-off frequency $f_{c}$ is eight times larger than the linear-operation Bragg frequency. As shown in [1], due to the diode losses, the number of Lvaractor cells should be reduced to as few as possible. For a realistic prediction of the maximum propagation frequency, a new technique based on harmonic balance (HB) is proposed here. This frequency $f_{\max }$ is defined as the input frequency from which an output voltage is below certain threshold. The value $V_{\text {out }}=10^{-4} \mathrm{~V}$ is considered here. To obtain $f_{\max }$ an auxiliary generator (AG) [8], operating at the input frequency $f_{A G}=f_{i n}$, is connected to the NLTL output node. The AG, with an ideal bandpass filter, must satisfy the non-perturbation condition given by the zero ratio between current and voltage $Y_{A G}=0$. For each input voltage $E_{i n}$, this condition is solved in terms of $\omega_{i n}$ and the AG phase $\phi_{A G}$. The number of harmonic terms considered is $N=40$. The application of this method to two different NLTL designs with $n=8$ cells, terminated in $50 \mathrm{Ohms}$, provides the results in Fig. 1(a). With a higher $n$, $f_{\max }$ decreases. Results for $L=0.2 \mathrm{nH}$ and $L=0.3 \mathrm{nH}$ are compared, obtaining higher $f_{\max }$ with a smaller $L$. The validity of the AG technique has been checked with independent timedomain and HB simulations $(N=40)$ [Fig. 1(b)]. Results for different $E_{\text {in }}$ and $L$ fully agree with the predictions of Fig. 1(a). Once $f_{\max }$ is known, the $10 \%-90 \%$ rise time can be roughly estimated from that of an RC low-pass filter, $\tau_{r}=0.35 / f_{\max }$. This approximate expression, applied to NLTLs in [4], must be used with caution. With $L=0.3 \mathrm{nH}$, the response to a ramp input is shown in Fig. 2(a), where the reduction of the wave rise time as it propagates through the NLTL cells can be noted. Oscillatory transients are obtained for input-ramp rise 
time smaller than $\pi\left(C_{o} L\right)^{1 / 2}$. In Fig. 2(b) a sinusoidal input has been considered. The output waveform shows a significant falling-edge compression. The waveform evolution through the various NLTL cells is represented in Fig. 2(c).
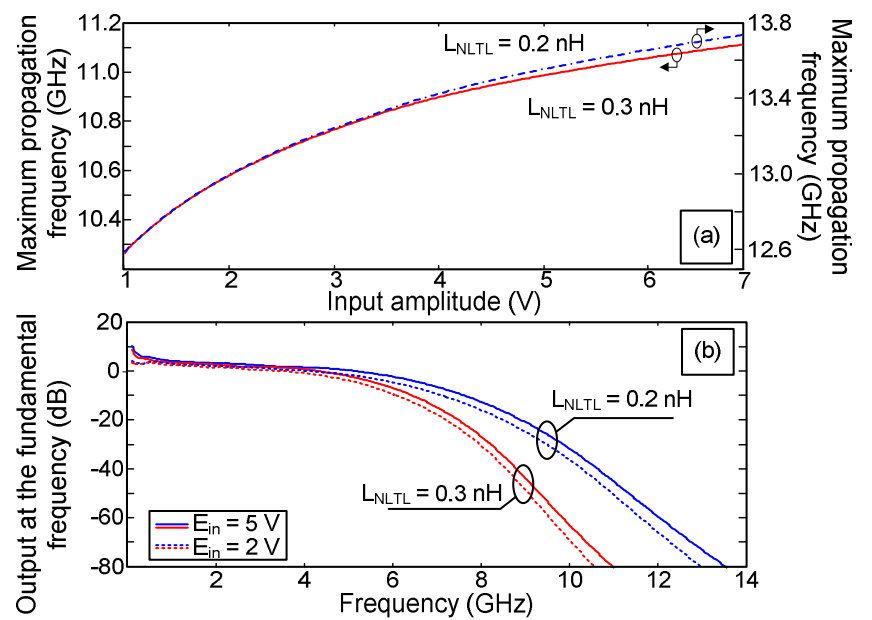

Fig. 1 AG technique to predict the maximum propagation frequency in an $8-$ cell realistic NLTL. (a) Variation of $f_{\max }$ vs. $E_{\text {in }}$ for two different $L$ values. (b) Validation with independent simulations.

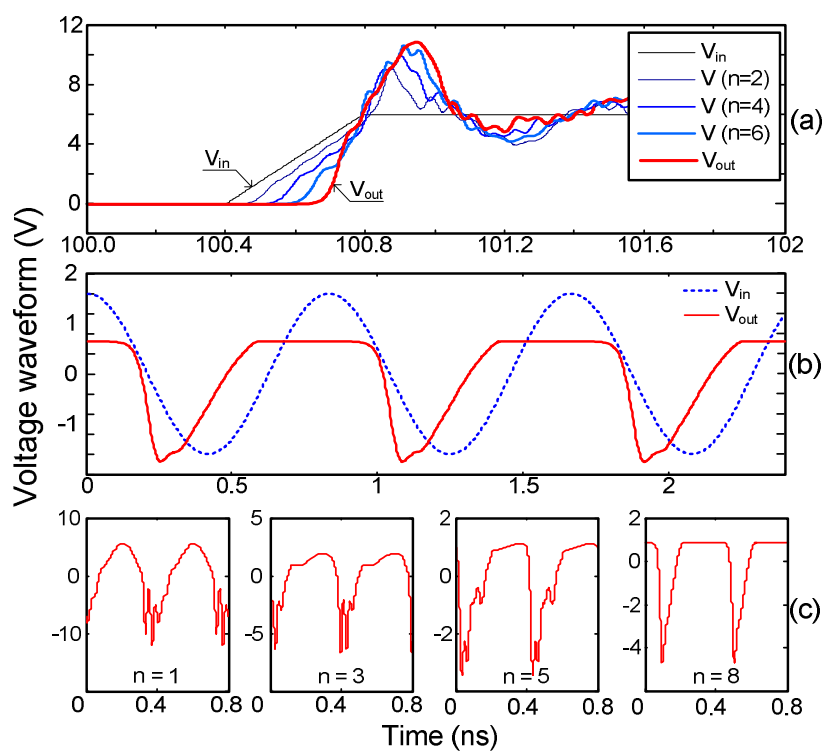

Fig. 2 NLTL with $n=8$ and $L=0.3 \mathrm{nH}$. (a) Response to a ramp input. (b) Sinusoidal input: comparison between input and output voltage. (c) Waveform evolution though the various NLTL cells.

To obtain the waveform-sharpening oscillator, a circuit capable to oscillate at the desired frequency $f_{o}=0.8 \mathrm{GHz}$ when loaded with the average NLTL characteristic impedance $\sqrt{L / C_{a v}}$ has been separately designed. It operates in near Class-E conditions [7] and includes a parallel feedback network to sustain the self-oscillation [Fig. 3(a)]. The series $L-C$ network at the transistor output enables the excitation of NLTL with a near-sinusoidal signal. For the full design optimization, a voltage $\mathrm{AG}$ at $f_{A G}=0.8 \mathrm{GHz}$ is connected in parallel at node 1 (Fig. 3). The AG amplitude is progressively increased until obtaining the desired waveform sharpening. The resulting output signal is shown in Fig. 4, where it can be compared with the one obtained with transient simulations. Connecting a grounded, high-impedance stub to the output node [Fig. 3(a)], it is possible to obtain a pulsed waveform through combination of reflection and delay effects. However, there is little control on the pulse amplitude and width. For more flexibility, a design based on the coupling of two waveform-sharpening oscillators will be studied.

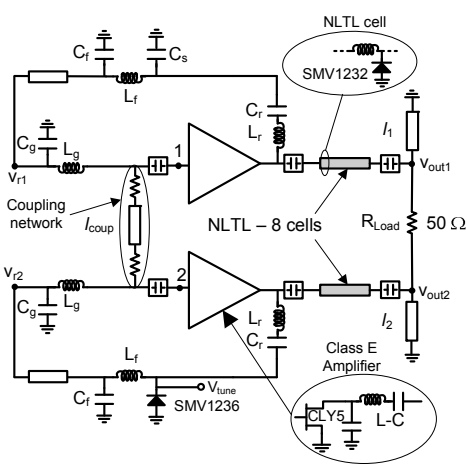

(a)

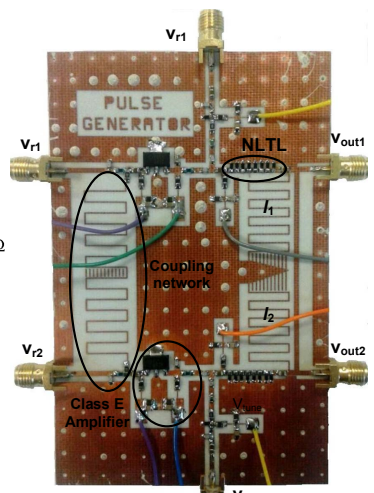

(b) 1$)^{v_{12}}$
Fig. 3 Pulse generator (2 coupled oscillators). (a) Schematic. (b) Photograph.

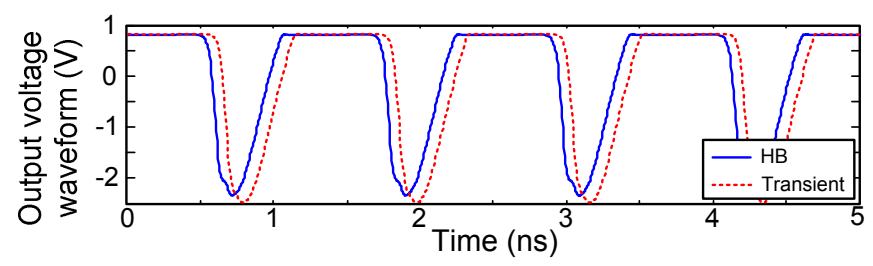

Fig. 4 Output signal of the sharpened-waveform oscillator terminated in $50 \Omega$.

\section{PULSE GENERATOR WITH TWO COUPLED OSCILLATORS}

The pulse generator is based on the coupling of two sharpening-waveform oscillators (Fig. 3). Due to the low NLTL characteristic impedance, the output signal can be taken in a differential manner $v_{\text {out }}(t)=v_{\text {out } 1}(t)-v_{\text {out } 2}(t)$, with a $50 \mathrm{Ohm}$ load between the NLTL output nodes. The coupling network, connected to the gate terminals (Fig. 3), consists of a transmission line bounded with series resistances [9]. The small current introduced into each oscillator will enable a mutual synchronization of the two oscillators. Three parameters will be available for the waveform synthesis: the two stub lengths $l_{1}$ and $l_{2}$ and the phase shift $\phi$, which is varied with a varactor diode connected to the feedback network of one of the oscillators [Fig. 3(a)]. Under weak coupling, this coupled operation will have negligible impact on the individual output waveforms. The three parameters $l_{1}, l_{2}$ and $\phi$ can be initially estimated in a separate analysis, exciting the two NLTLs (each terminated in a different grounded stub) with phase shifted sinusoidal sources, of the same amplitude as the oscillator at the NLTL connection point. The varactorbias voltage $\Delta \eta_{1}$ for a particular $\phi$ can be estimated with a reduced-order model, obtained through linearization of each oscillator admittance function about the free-running solution. This is carried out with an AG, introduced in parallel at the 
node to which the coupling network will be connected (nodes 1 and 2 in Fig. 1). The admittance-function derivatives with respect to amplitude $Y_{V}$, frequency $Y_{\omega}$ and bias voltage $Y_{\eta}$ are calculated through finite differences [8]. The admittance matrix of the symmetric coupling network has element values $Y_{11}=Y_{22}=Y_{e}$ and $Y_{12}=Y_{21}=Y_{n b}$. Applying Kirchoff's laws [8], the two coupled-oscillator equations are:

$$
\begin{aligned}
& {\left[Y_{V} \Delta V_{1}+Y_{\omega} \Delta \omega+Y_{\eta} \Delta \eta_{1}\right]=-Y_{e}-Y_{n b} e^{j \phi}} \\
& {\left[Y_{v}^{\prime} \Delta V_{2}+Y_{\omega}^{\prime} \Delta \omega\right]=-Y_{n b} e^{-j \phi}-Y_{e}}
\end{aligned}
$$

where different amplitude and frequency derivatives have been assumed and second-order terms have been neglected. In general, the output grounded stub, located far from the oscillator coupling point, will have negligible influence on the derivatives. Then, $\Delta \eta_{1}$ and $\Delta \omega$ are estimated from:

$$
\begin{aligned}
& \Delta \eta_{1}=-\frac{2\left|Y_{n b}\right| \cos \alpha_{v, n b} \sin \phi}{\left|Y_{\eta}\right| \sin \alpha_{v, \eta}} \\
& \Delta \omega=-\frac{\left|Y_{e}\right| \sin \alpha_{v, e}}{\left|Y_{\omega}\right| \sin \alpha_{v, \omega}}-\frac{\left|Y_{n b}\right|}{\left|Y_{\omega}\right| \sin \alpha_{v, \omega}} \sin \left(\alpha_{v, n b}-\phi\right)
\end{aligned}
$$

In case derivatives are very different, one can directly solve system (3). The qualitative behavior is quite different from the one obtained in [8] with 3 oscillator elements. The tuning voltage $\Delta \eta_{1}$ varies with $\phi$ in sinusoidal manner. The oscillation frequency undergoes a small constant offset due to loading effects and has zero deviation from this offset at $\phi=\alpha_{v, n b}$. To prevent any variation of $f_{o}$ at fixed $\phi_{o}$, the length $l_{\text {coup }}$ of the coupling line can be swept, (thus modifying $Y_{e}$ and $Y_{n b}$ ) to select the value that provides $\Delta \omega=0$. With the separate simulation of the differential NLTL, the parameter values for the narrowest pulse are $\phi=25^{\circ}, l_{1}=181^{\circ}$ and $l_{2}=182^{\circ}$. From (4)(a), $\phi=25^{\circ}$ corresponds to $\Delta \eta_{1}=0.5 \mathrm{~V}$. Using this value in a full $\mathrm{HB}$ simulation of the coupled oscillators, one obtains the waveform in Fig. 5(a). Stable behavior has been verified. The measured waveform is on the right side. In both simulation and measurement, the pulse is compared with the narrowest output pulse of a single oscillator. This pulse duty cycle is $7 \%$, whereas in the work [7], based on soliton propagation, it is $10 \%$. The simulated and measured waveforms of the individual-oscillator outputs (forming the pulse) are shown in Fig. 5(b). With $l_{1}=l_{2}=181^{\circ}$ and $\phi=15^{\circ}$, a monocycle is obtained. From (4)(a), the tuning voltage required is $\Delta \eta_{1}=0.3 \mathrm{~V}$. The $\mathrm{HB}$ simulation and measurement results are shown in Fig. 5(c). Discrepancies are attributed to parasitics in the hybrid NLTL. At $100 \mathrm{kHz}$, the measured phase-noise spectral density of the individual oscillator is $\mathrm{S}(\mathrm{f})=-91.23 \mathrm{dBc} / \mathrm{Hz}$, whereas for the coupled oscillator it is smaller: $\mathrm{S}(\mathrm{f})=-94.3 \mathrm{dBc} / \mathrm{Hz}$.

\section{CONCLUSION}

An analysis and design methodology for pulse generation based on the shock-wave concept in NLTL has been presented. The maximum propagation frequency through the NLTL is estimated with a realistic numerical technique. The coupling of two NLTL oscillators terminated in different stubs provides a flexible design. The required tuning voltage and the frequency deviation with respect to free-running conditions are predicted with a reduced-order model.
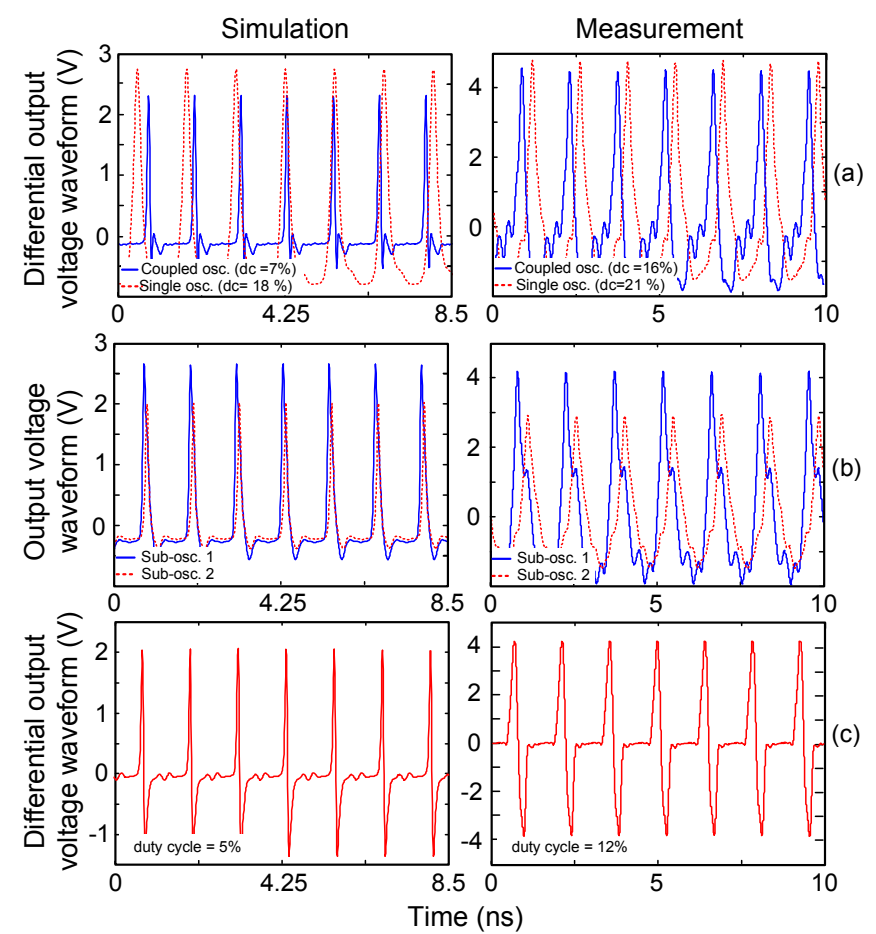

Fig. 5 Simulated (left) and measured (right) waveforms of the coupled oscillators. (a) Pulse obtained for $l_{1}=181^{\circ}, l_{2}=182^{\circ}$ and $\phi=25^{\circ}$. (b) Waveforms that conform the pulse in (b). (c) Monocycle for $l_{1}=l_{2}=181^{\circ}$ and $\phi=15^{\circ}$.

\section{ACKNOWLEDGEMENT}

Spanish project TEC2011-29264-C03-01 for financial support.

\section{REFERENCES}

[1] F. Martín, X. Oriols, J. A. Gil, J. García-García, "Optimization of Nonlinear Transmission Lines for Harmonic Generation," Int. Jour. of Infrared and Millimeter Waves, vol. 23, no. 1, pp. 95-103, Jan., 2002.

[2] E. Afshari, A. Hajimiri, "Nonlinear Transmission Lines for Pulse Shaping in Silicon," IEEE J. Solid-State Circ., vol. 40, no. 3, pp. 744-752, 2005.

[3] M. Remoissenet, Waves Called Solitons: Concepts and Experiments, New York: Springer, 1999.

[4] M.Rodwell, D.M.Bloom, B.A. Auld, "Nonlinear transmission line for picosecond pulse compression and broadband phase modulation," Electronic Letters, vol. 23, no. 3, pp. 109-110, Jan., 1997.

[5] M. Rodwell et al. "GaAs Nonlinear transmission lines for picosecond pulse generation and millimetre wave sampling," IEEE T-MTT, vol. 39, no. 7, pp: 1194-2004, 1991.

[6] D.S. Ricketts, X.Li, N. Sun, K.Woo, D.Ham, "On the Self-Generation of Electrical Soliton Pulses," IEEE J. Solid-State Circuits, vol. 42, no. 8, pp. 1657-1668, Aug., 2007.

[7] M. Pontón, E. Fernández, F. Ramírez, A. Suárez, "Optimized Design of Pulsed Waveform Oscillators and Frequency Dividers", IEEE T-MTT, vol. 59, no. 12, pp. 3428-3440, 2011.

[8] A. Suárez, F. Ramírez, S. Sancho, "Stability and Noise Analysis of Coupled-Oscillator Systems," IEEE T-MTT, vol. 59, no. 4, pp. 10321046, 2011.

[9] R. A. York, "Nonlinear analysis of phase relationships in quasi-optical oscillator arrays," IEEE T-MTT, vol. 41, no. 10, pp. 1799 - 1809, 1993. 